\title{
Diagnostic Performance of HPV E6/E7 mRNA and HPV DNA Assays for the Detection and Screening of Oncogenic Human Papillomavirus Infection among Woman with Cervical Lesions in China
}

\author{
Hye-young Wang ${ }^{1}$, Dongsup Lee ${ }^{2,3}$, Sunyoung Park ${ }^{2}$, Geehyuk Kim², Sunghyun \\ $\mathrm{Kim}^{4}$, Lin Han ${ }^{5}$, Ren Yubo ${ }^{5}$, Yingxue $\mathrm{Li}^{5}$, Kwang Hwa Park ${ }^{6 *}$, Hyeyoung Lee ${ }^{2 *}$
}

\begin{abstract}
Background: Human papillomavirus (HPV) is the most common sexually transmitted infection worldwide and it is responsible for most cases of cervical uterine cancer. Although HPV infections of the cervix do not always progress to cancer, $90 \%$ of cervical cancer cases have been found to be associated with high risk HPV (HRHPV) infection. HPV DNA testing is widely used, along with Papanicolaou (Pap) testing, to screen for cervical abnormalities. However, there are no data on the prevalence of genotype-specific HPV infections assessed by measuring HPV E6/E7 mRNA in women representative of the Chinese population across a broad age range. Materials and Methods: In the present study, we compared the results with the CervicGen HPV RT-qDx assay, which detects 16 HR-HPV genotypes (Alpha-9: HPV 16, 31, 33, 35, 52, and 58; Alpha-7: HPV 18, 39, 45, 51, 59, and 68; and Alpha-5, 6: HPV 53, 56, 66, and 69), and the REBA HPV-ID assay, which detects 32 HPV genotypes based on the reverse blot hybridization assay (REBA) for the detection of oncogenic HPV infection according to cytological diagnosis. We also investigated the prevalence and genotype distribution of HPV infection with a total of 324 liquid-based cytology samples collected in western Shandong province, East China. Results: The overall HPV prevalences determined by HPV DNA and HPV E6/E7 mRNA assays in this study were $79.9 \%(259 / 324)$ and 55.6\% (180/324), respectively. Although the positivity of HPV E6/E7 mRNA expression was significantly lower than HPV DNA positivity, the HPV E6/E7 mRNA assay showed greater specificity than the HPV DNA assay (88.6\% vs. 48.1\%) in normal cytology samples. The prevalence of Alpha-9 (HPV 16, 31, 33, 35, 52, and 58) HPV infection among these women accounted for up to $80.3 \%$ and $76.1 \%$ of the high-grade lesions detected in the HPV mRNA and DNA tests, respectively. The HR-HPV genotype distribution, based on HPV DNA and E6/E7 mRNA expression by age group in patients with cytologically confirmed lesions, was highest in women aged 40 to 49 years $(35.9 \%$ for cytologically confirmed cases, Pearson correlation $r$ value $=0.993, p<0.001)$ for high-grade lesions. Among the oncogenic HR-HPV genotypes for all age groups, there was little difference in the distribution of HPV genotypes between the HPV DNA (HPV -16, 53, 18, 58, and 33) and HPV E6/E7 mRNA (HPV -16, 53, 33, 58, and 18) assays. HPV 16 was the most common HPV genotype among women with highgrade lesions. Conclusions: Our results suggest that the HPV E6/E7 mRNA assay can be a sensitive and specific tool for the screening and investigation of cervical cancer. Furthermore, it may provide useful information regarding the necessity for early cervical cancer screenings and the development of additional effective HPV vaccines, such as one for HPV 53 and 58 . Additionally, gaining knowledge of HPV distribution may also inform us about ecological changes in HPV after the vaccination.
\end{abstract}

Keywords: Cervical cancer - HPV - E6/E7 mRNA - REBA - prevalence - molecular diagnosis

Asian Pac J Cancer Prev, 16 (17), 7633-7640

\section{Introduction}

Human uterine cervical cancer is the third most common cancer among women worldwide (Jemal et al.,
2011) and a major cause of morbidity and mortality. In China, the most populous country in the world, cervical cancer remains the second leading cause of cancer death among 15- to 44-year-old females (Sanjose et al., 2012).

${ }^{1}$ Wonju Eco Environmental Technology Center, ${ }^{2}$ Department of Biomedical Laboratory Science, College of Health Sciences, Yonsei University, Wonju, Gangwon, Department of Clinical Laboratory Science, ${ }^{3}$ Hyejeon College, Hongseong, Chungnam, ${ }^{4}$ College of Health Sciences, Catholic University of Pusan, Busan, Department of Pathology, Republic of Korea ${ }^{5}$ Liaocheng School of Clinical Medicine, Taishan Medical University, Liaocheng, Shandong, China, ${ }^{6}$ Yonsei University Wonju College of Medicine, Wonju, Gangwon, Republic of Korea, *For correspondence: Kwang Hwa Park,abba@yonsei.ac.kr,Hyeyoung Lee, hyelee@yonsei.ac.kr 
In particular, there are 75,500 new cases and 34,000 deaths annually in developing countries such as China (Xiao-Xiang et al., 2014).

Human papillomavirus (HPV) is the most common sexually transmitted pathogen among women and men, and HPV infection is considered to be the main contributing factor in the development of cervical cancer (Walboomers et al., 1999). It has been estimated that $70 \%$ of sexually active women will acquire an HPV infection at some point during their lifetime (Walboomers et al., 1999). Approximately $60 \mathrm{HPV}$ genotypes are known to infect the genital tract (Ergünay et al., 2007), and 15 genotypes (including HPV 16, 18, 33, 45, 52, 58, 39, 51, 56, and 59) are classified as HR-HPV genotypes. The majority of HPVs belong to the genus alpha-papilloma virus, which can be further subdivided into species and strains. Among these, alpha-7 (HPV-18, -39, -45, -59, -68) and alpha-9 (HPV-16, -31, -33, -35, -52, -58) are related to high oncogenic risk (Ault, 2007). Oncogenic HPV genotypes cause invasive cervical carcinoma or squamous intraepithelial lesions upon infection of cervical epithelial cells (zur Hausen, 2009). As a result, testing for oncogenic HPV infection in cervical lesions could serve as an accurate means of identifying women who are at risk for developing cervical cancer. In general, HPV types 16 and 18 cause approximately $70 \%$ of cervical cancers and types $31,33,35,45,52$, and 58 accounts for an additional $20 \%$ of cases (Clifford et al., 2005). However, the prevalence of HPV infection and type-specific distribution varies depending on the country and province, and substantial geographical variation are known to exist (Bruni et al., 2010; Poljak et al., 2013).

The incidence of uterine cervical cancer has been decreasing since the implementation of periodic cancer screening programs which detect cervix dysplasia and in situ carcinoma. The utilization of the cervix Papanicolaou smear (Pap smear) has been a key reason for the decrease in cervical cancer incidence rates and the accompanying mortality (Okadome et al., 2014). Although the Pap smear has been a widely used cervical screening test due to its low cost and simple procedure, one main drawback is the false negative error rate of 20\%-50\%. In addition, the sensitivity for uterine cervix high-grade squamous intraepithelial lesion detection and the predictive value have been questioned by previous reports (Vizcaino et al., 1998). The progression of a precancerous lesion to an invasive carcinoma in uterine cervical cancer occurs slowly. Therefore, early detection is the most effective way of halting this progression. As a supplement to the Pap smear, the HPV molecular diagnosis test is effective in diagnosing HR-HPV groups in cervical cancer.

Several PCR-based multiplex detection methodologies have been shown to be effective in detecting HPV infections, and clinicians and researchers have used PCRbased molecular assays (Laudadio, 2013). For uterine cervical cancer diagnosis, most molecular diagnostics are based on an HPV DNA test with Pap smear. Although the sensitivity of HPV DNA testing is good, its specificity is relatively low. Currently, European countries such as Norway and Finland recommend the guidelines for follow-up exams for cervical examination and HPV E6/
E7 mRNA testing (Sveinung et al., 2010). Although it has been reported that the HPV E6/E7 mRNA test has greater specificity for detecting cancer development than do L1 gene-based DNA tests (Sveinung et al., 2010; Andersson et al., 2011), there are no data on the distribution of typespecific HPV infections among HPV mRNA detected in women representative of the Chinese population covering a broad age range.

In the present study we used the CervicGen HPV RT-qDx assay (Optipharm, Osong, Republic of Korea), which targets HPV E6/E7 mRNA of 16 oncogenic HR HPV genotypes $(16,18,31,33,35,39,45,51,52,53$, $56,58,59,66,68$, and 69$)$ to investigate the prevalence and genotype distribution in relation to age in Chinese women, cytology of oncogenic HPV infection compared with the REBA HPV-ID which is based on the reverse blot hybridization assay (REBA).

\section{Materials and Methods}

\section{Clinical samples}

Liquid-based cytology samples (Table 1) from 326 women were obtained over a 1-year (from January to December 2013) period from the Liaocheng People's Hospital in the Shandong province of China. This study was approved by the Institutional Ethics Committee at Liaocheng People's Hospital (approval no. 2011-0038), and all subjects provided written informed consent. All clinical samples were collected using ThinPrep ${ }^{\circledR}$ (Hologic Inc., Bedford, MA, USA) Pap materials.

\section{Cytological and histological diagnosis}

Liquid-based cytology slides (Pap smears) were evaluated according to the 2001 Bethesda System designed by cytopathologists and pathologists. Cytological cases of squamous cell carcinoma (SCC), high-grade squamous intraepithelial lesion (H-SIL), atypical squamous cells cannot exclude H-SIL (ASC-H), a low-grade squamous intraepithelial lesion (L-SIL), typical squamous cells of undetermined significance (ASC-US), and normal (within normal limit, include reactive change due to inflammation, fungal infection, and atrophy) were included. The remaining fluid samples were stored at $4^{\circ} \mathrm{C}$ after cytology slide preparation and prior to DNA extraction.

\section{PCR-reverse blot hybridization assay (PCR-REBA) for $H P V$ genotyping}

HPV genotyping assay was performed using the REBA HPV-ID ${ }^{\circledR}$ test (YD Diagnostic, Yongin, Republic of Korea) which is based on PCR-REBA and was performed according to the manufacturer's instructions. The REBA HPV-ID is designed to detect $18 \mathrm{HR}-\mathrm{HPV}$ genotypes (16, $18,26,31,33,35,39,45,51,52,53,56,58,59,66,68$, 69 , and 73) together with 14 low-risk (LR) HPV genotypes $(6,11,32,34,40,42,43,44,54,70,72,81,84$, and 87). PCR was performed using a $20-\mu \mathrm{L}$ reaction mixture (Genetbio, Daejeon, Republic of Korea) containing $2 \times$ master mix, $1 \times$ primer mixture, $5 \mu \mathrm{L}$ sample DNA, and sterile distilled water (DW) to give a final volume of 20 $\mu \mathrm{L}$. The first 10 PCR cycles consisted of denaturation at $95^{\circ} \mathrm{C}$ for $30 \mathrm{~s}$, followed by annealing and extension 
at $60^{\circ} \mathrm{C}$ for $30 \mathrm{~s}$. These 10 cycles were followed by 40 cycles of denaturation at $95^{\circ} \mathrm{C}$ for $30 \mathrm{~s}$ and annealing and extension at $54^{\circ} \mathrm{C}$ for $30 \mathrm{~s}$. After the final cycle, samples were maintained at $72^{\circ} \mathrm{C}$ for 10 min to complete synthesis of all strands. The amplified target was visualized as a single band corresponding to a length of $150 \mathrm{bp}$ using the Chemi Doc system (Vilber Lourmat, Deutschland, Germany).

For the REBA HPV-ID ${ }^{\circledR}$, the hybridization and washing processes were performed according to the manufacturer's instructions. In brief, the biotinylated PCR products were denatured at $25^{\circ} \mathrm{C}$ for $5 \mathrm{~min}$ in denaturation solution and diluted in $970 \mu \mathrm{L}$ of hybridization solution on the REBA membrane strip in the provided blotting tray. Denatured single-stranded PCR products were hybridized to the probes on a strip at $50^{\circ} \mathrm{C}$ for $30 \mathrm{~min}$. The strips were then washed twice with gentle shaking in $1 \mathrm{~mL}$ of washing solution for $10 \mathrm{~min}$ at $50^{\circ} \mathrm{C}$, incubated at $25^{\circ} \mathrm{C}$ with streptavidin-alkaline phosphatase (AP) conjugate (Roche Diagnostics, Mannheim, Germany) diluted 1:2,000 in conjugate diluents solution (CDS) for $30 \mathrm{~min}$, and finally washed twice with $1 \mathrm{mLCDS}$ at room temperature for $1 \mathrm{~min}$. The colorimetric hybridization signals were visualized by addition of a 1:50 dilution of AP-mediated staining solution, NBT/BCIP (Roche Diagnostics), and incubation was continued until a color change was detected. Finally, the banding pattern was read and interpreted.

\section{Total RNA isolation}

After cytology slide preparation was complete, the remainder of the specimen was used for RNA isolation. Total cellular RNA was isolated using the Isol-RNA Lysis Reagent (5 Prime, Austin, TX, USA) according to the manufacturer's instructions. Briefly, $1 \mathrm{~mL}$ of Isol-RNA Lysis Reagent (5 Prime) was added to the cellular pellet. Cells were lysed by vortexing or repeated pipetting, and left to stand at room temperature for $5 \mathrm{~min}$. Subsequently, $200 \mu \mathrm{L}$ of chloroform was added and the mixture was shaken vigorously and incubated at room temperature for $3 \mathrm{~min}$ before centrifugation at $12,000 \times \mathrm{g}$ for $15 \mathrm{~min}$. The resultant aqueous layer was transferred to a new tube and an equal volume of isopropanol was added and mixed by inverting the tube. After incubation for $10 \mathrm{~min}$ at $25^{\circ} \mathrm{C}$ and centrifugation at $12,000 \times \mathrm{g}$ for 10 min, $1 \mathrm{~mL}$ of $75 \%$ ethanol was added to the supernatant and mixed by tube inversion. Finally, the mixture was centrifuged at 7,500 $\times \mathrm{g}$ for $5 \mathrm{~min}$, and the supernatant was removed. The RNA pellet was dried and eluted in 30 $\mu \mathrm{L}$ of diethylpyrocarbonate (DEPC)-treated water (Intron Biotechnology, Seoul, Republic of Korea). The purity and concentration of total RNA were determined by measuring the absorbance at 260 and $280 \mathrm{~nm}$ using the Infinite $200 \AA$ (Tecan, Salzburg, Austria). All steps in the preparation and handling of total RNA were performed in a laminar flow hood under RNase-free conditions. The isolated total RNA was stored at $-70^{\circ} \mathrm{C}$.

\section{cDNA synthesis}

Complementary DNA (cDNA) was synthesized using an M-MLV Reverse Transcriptase kit (Invitrogen,
Carlsbad, CA, USA) and random hexamers (Invitrogen), according to the manufacturer's recommendations. Briefly, $10 \mu \mathrm{L}$ of total RNA was added to a master mix containing $1 \mu \mathrm{L} 10 \mathrm{mM}$ dNTP mix (10 mM each dATP, $\mathrm{dGTP}, \mathrm{dCTP}$, and dTTP at a neutral $\mathrm{pH}), 0.25 \mu \mathrm{g}$ random hexamers, and $5 \mu \mathrm{L}$ DEPC-treated water in PCR tubes. The reaction mixture was incubated at $65^{\circ} \mathrm{C}$ for $5 \mathrm{~min}$ and quickly chilled on ice. After addition of $4 \mu \mathrm{L} 5 \times$ FirstStrand Buffer, $2 \mu \mathrm{L} 0.1 \mathrm{M}$ dithiothreitol (DTT), and $1 \mu \mathrm{L}$ M-MLV reverse transcriptase (RT), the cDNA synthesis reaction was performed at $25^{\circ} \mathrm{C}$ for $10 \mathrm{~min}, 37^{\circ} \mathrm{C}$ for 50 $\min$, and $70^{\circ} \mathrm{C}$ for $15 \mathrm{~min}$.

\section{HPV E6/E7 mRNA RT-qPCR assay}

Detection of HPV E6/E7 mRNA in cervical specimens was performed by quantitative reverse-transcriptase-PCR (RT-qPCR). The RT-qPCR TaqMan assay was carried out with the CervicGen HPV RT-qDx assay kit (Optipharm) using CFX-96 (Bio-Rad, Hercules, CA, USA) and ABI 7500 Fast (Applied Biosystems, Foster City, CA, USA) real-time PCR systems for thermocycling and fluorescence detection, according to the manufacturers' instructions. The PCR primers and the corresponding TaqMan ${ }^{\circledR}$ probes were designed for three different sets of HPVs, in each case targeting their common sequence (Alpha-9: HPV genotypes 16, 31, 33, 35, 52, and 58; Alpha-7: HPV genotypes 18, 39, 45, 51, 59, and 68; and Alpha-5,6: HPV genotypes 53, 56, 66, and 69). Real-time PCR amplification for HPV E6/E7 mRNA was performed in a total volume of $25 \mu \mathrm{L}$ containing $12.5 \mu \mathrm{L} 2 \times$ Thunderbird probe qPCR mix (Toyobo, Osaka, Japan), $7.5 \mu \mathrm{L}$ primer and TaqMan probe mixture, $5 \mu \mathrm{L}$ template cDNA, and DW to give a final volume of $25 \mu \mathrm{L}$ for each sample. The multiplex RT-qPCR assay detected HPV E6/E7 genes simultaneously in a single tube by incorporating 3 target-specific TaqMan probes, which were labeled with different fluorophores (FAM, HEX, and Cy5). Positive and negative controls were included throughout the procedure. No-template controls with sterile DW instead of template DNA were incorporated into each run. Cycling conditions were $95^{\circ} \mathrm{C}$ for $3 \mathrm{~min}$, followed by 45 cycles of $95^{\circ} \mathrm{C}$ for $20 \mathrm{~s}$ and $60^{\circ} \mathrm{C}$ for $40 \mathrm{~s}$. The mRNA expression level was quantified by determining the cycle threshold (CT), which is the number of PCR cycles required for the fluorescence to exceed a value significantly higher than the background fluorescence. To avoid false negatives due to degradation of mRNA, glyceraldehyde-3-phosphate dehydrogenase (GAPDH) was used as a control. Target gene mRNA expression levels relative to GAPDH were automatically calculated using the comparative $\mathrm{Ct}$ method by CFX Manager Software v1.6 (Bio-Rad).

\section{Statistical analysis}

All statistical analyses were performed using Prism 5 software (GraphPad, La Jolla, CA, USA) and SPSS statistics software version 21.0 (IBM, Armonk, NY, USA). Sensitivity, specificity, correlation $\mathrm{p}$ value, and $95 \%$ confidence interval (CI) of the predictive ability for each HPV DNA chip or REBA HPV-ID ${ }^{\circledR}$ and CervicGen HPV RT-qDx assay were estimated with respect to cytological and histological diagnosis. 


\section{Results}

Cytological diagnosis for liquid-based cytology specimens

A total of 324 specimens obtained from Chinese women were classified into six categories by cytological criteria: $4(1.2 \%)$ as SCC, $67(20.7 \%)$ as HSIL, 19 (5.9\%) as ASC-H, 59 (18.2\%) as LSIL, 96 (29.6\%) as ASC-US, and $79(24.4 \%)$ as normal (Table 1). The age range of patients was 21 to 79 years, with a mean age of 43.4 years $(\mathrm{SD} \pm 12.6$ years, $95 \%$ confidence interval $(\mathrm{CI})$ 42.6-45.3). Among the 324 cytology specimens, samples from Chinese women who were $<30$ years old, 30-39 years old, 40-49 years old, 50-59 years old and $\geq 60$ years old accounted for 50 (15.4\%), 64 (19.8\%), 97 (29.9), 77 $(23.8 \%)$ and $36(11.1 \%)$ of the samples, respectively (Table 1).

Prevalence rates of the HPV DNA and HPVE6/E7 mRNA assays in cytological diagnosis

The prevalence of each HR-HPV group was classified according to Alpha-9 (HPV genotypes 16, 31, 33, 35, 52, and 58), Alpha-7 (HPV genotypes 18, 39, 45, 51, 59, and 68), and Alpha-5, 6 (HPV genotypes 53, 56, 66, and 69) species based on HPV DNA and HPV E6/E7 mRNA expression in 324 cytologically diagnosed specimens. The distribution of these groups was analyzed within HPV positive samples including mixed infections. In the HPV DNA assay, the prevalence rates of HR-HPV Alpha-9 were $2(50.0 \%)$ in SCC, $55(84.6 \%)$ in HSIL, $19(90.4 \%)$ in ASC-H, 17 (38.6\%) in LSIL, 50 (52.6\%) in ASCUS, and $5(50.0 \%)$ in normal. In the HR-HPV Alpha-7 group, we found that $1(25.0 \%)$ in SCC, $2(3.1 \%)$ in HSIL, $1(4.8 \%)$ in ASC-H, 11 (25.0\%) in LSIL, 7 (18.4\%) in ASCUS, and $4(40.0 \%)$ in normal were HPV DNA positive. For HRHPV Alpha-5, 6, 1 (25.0\%) in SCC, 8 (12.3\%) in HSIL, $1(4.8 \%)$ in ASC-H, 16 (36.4\%) in LSIL, 11 (29.0\%) in ASCUS, and $1(10.0 \%)$ in normal were found to be HPV DNA positive (Figure 1A).

In the HPV E6/E7 mRNA assay, the prevalence rates of HR-HPV Alpha-9 were 4 (66.7\%) for SCC, 56 (77.1\%) for HSIL, 18 (74.1\%) for ASC-H, 23 (28.9\%) for LSIL, $31(26.4 \%)$ for ASCUS, and $7(6.9 \%)$ for normal. For the HR-HPV Alpha-7 group, 1 (16.6\%) for SCC, 3 (4.1\%) for HSIL, 1 (4.1\%) for ASC-H, $20(25.1 \%)$ for LSIL, 17 (14.5\%) for ASCUS, and 1 (1.0\%) for normal were found to be HPV E6/E7 mRNA positive. For HR-HPV Alpha-5, 6, 1 (16.6\%) for SCC, 5 (6.9\%) for HSIL, 4 (16.5\%) for ASC-H, 15 (18.9\%) for LSIL, 12 (10.2\%) for ASCUS, and $1(1.0 \%)$ for normal were determined to be HPV E6/ E7 mRNA positive (Figure 1B).

Table 1. Clinical samples used in this study
Comparison of the positivity rates determined by $\mathrm{HPV}$ DNA and HPV E6/E7 mRNA assays

The positivity rates of HPV E6/E7 mRNA expression were compared with HPV DNA based on cytological diagnosis. The overall HPV prevalences determined using HPV DNA and the HPV E6/E7 mRNA assay in this study were $79.9 \%(259 / 324)$ and $55.6 \%$ (180/324), respectively (Table 2). The HPV DNA positivity was $4 / 4$ $(100 \%)$ in cases of SCC , 62/67 $(92.5 \%)$ for HSIL cases, $18 / 19(94.7 \%)$ for cases of ASC-H, 55/59 (93.2\%) for LSIL cases, 79/96 (82.3\%) for cases of ASC-US, and $41 / 79$ (51.9\%) for normal. The HR-HPV prevalences

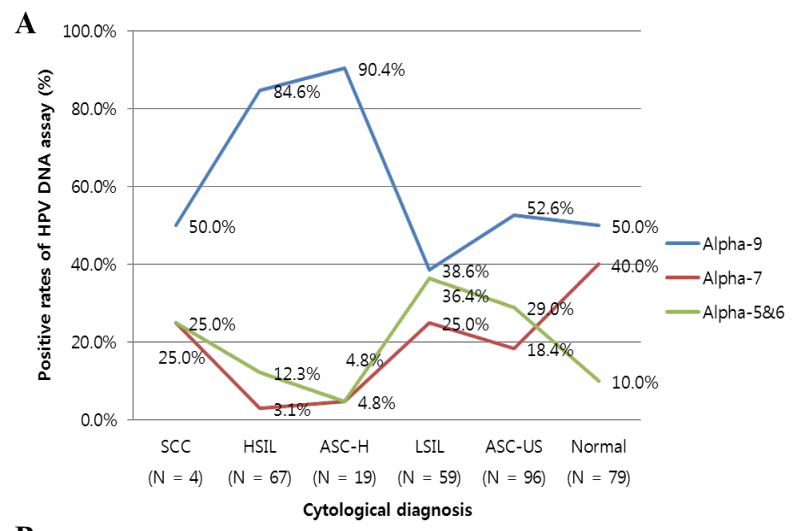

B

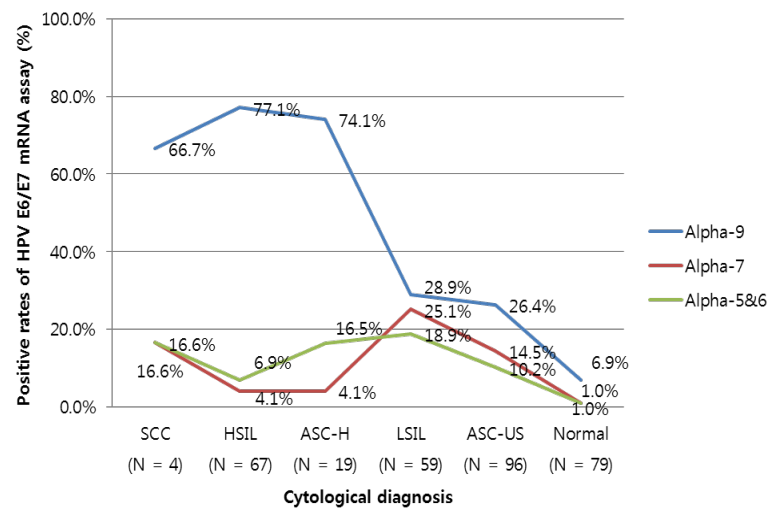

Figure 1. Overall Prevalence of HR-HPV Alpha-9, Alpha-7, and Alpha-5,6 Determined by HPV DNA (A) and HPV E6/E7 mRNA (B) assays based on cytological diagnosis from Chinese subjects. The HR-HPV group prevalence was determined by Alpha-9 (HPV genotypes 16, $31,33,35,52$, and 58), Alpha-7 (HPV genotypes 18, 39, 45, 51, 59, and 68), and Alpha-5, 6 (HPV genotypes 53, 56, 66, and 69) species based on HPV E6/E7 mRNA expression in 324 cytologically diagnosed specimens. Abbreviations: SCC, squamous cell carcinoma; H-SIL, high grade squamous intraepithelial lesion; ASC-H, atypical squamous cells - cannot exclude H-SIL; LSIL, low grade squamous intraepithelial lesion; ASCUS, atypical squamous cells of undetermined significance.

\begin{tabular}{lllccrrrr}
\hline Ethnicity & Cytological & Median & \multicolumn{5}{c}{ Age group } & Totaln (\%) \\
\cline { 5 - 7 } & diagnosis & age & $<30, \mathrm{n}(\%)$ & $30 \sim 39, \mathrm{n}(\%)$ & $40 \sim 49, \mathrm{n}(\%)$ & $50 \sim 59, \mathrm{n}(\%)$ & $\geq 60, \mathrm{n}(\%)$ & \\
\hline Chinese & SCC & 56 & $0(0)$ & $0(0)$ & $0(0)$ & $3(0.9)$ & $1(0.3)$ & $4(1.2)$ \\
$(\mathrm{n}=324)$ & HSIL & 44 & $8(2.5)$ & $13(6.3)$ & $24(7.4)$ & $16(4.9)$ & $6(1.9)$ & $67(20.7)$ \\
& ASC-H & 44 & $1(0.3)$ & $3(0.9)$ & $8(2.5)$ & $6(1.9)$ & $1(0.3)$ & $19(5.9)$ \\
& LSIL & 36 & $16(4.9)$ & $16(4.9)$ & $14(4.3)$ & $8(2.5)$ & $5(1.5)$ & $59(18.2)$ \\
& ASC-US & 45.5 & $10(3.1)$ & $15(4.6)$ & $28(8.6)$ & $27(8.3)$ & $16(4.9)$ & $96(29.6)$ \\
& normal & 42 & $15(4.6)$ & $17(5.2)$ & $23(7.1)$ & $17(5.2)$ & $7(2.2)$ & $79(24.4)$ \\
& Total & 43.4 & $50(15.4)$ & $64(19.8)$ & $97(29.9)$ & $77(23.8)$ & $36(11.1)$ & $324(100)$ \\
\hline
\end{tabular}


Table 2. Prevalence of oncogenic HPV infection determined by HPV DNA and HPV E6/E7 mRNA assays among women with cytological lesions

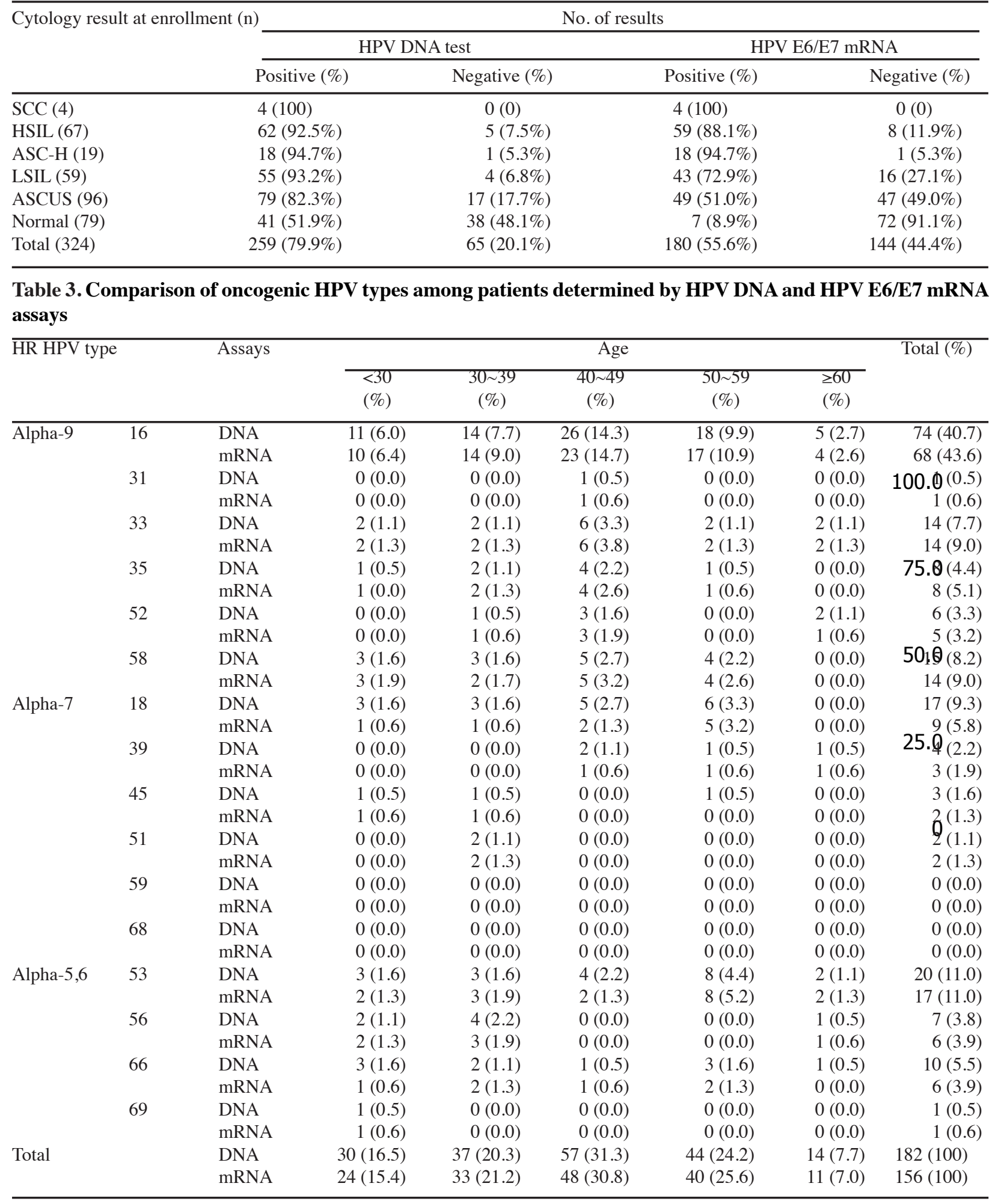

among all cytological grade samples were $162(50.5 \%)$ and 4/4 (100\%) for SCC, 58/67 (86.6\%) for HSIL, 17/19 $(89.5 \%)$ for ASC-H, 39/59 (66.1\%) for LSIL, 33/96 (34.4\%) for ASC-US, and 11/79 (13.9\%) for normal. All 324 liquid based cytology specimens were positive for GAPDH mRNA expression. Among the 324 cytologically diagnosed specimens, HPV E6/E7 mRNA positivity was detected in 4/4 (100\%) SCC samples, 59/67 (88.1\%) HSIL samples, 18/19 (94.7\%) ASC-H samples, 43/59 (72.9\%) LSIL samples, 49/96 (51.0\%) ASCUS samples, and 7/79
$(8.9 \%)$ normal samples (Figure 2A).

Comparison of HPV genotypes based on L1 gene target DNA and E6/E7 mRNA analysis

The DNA genotypes and E6/E7 mRNA defined according to the Alpha-9, Alpha-7 and Alpha-5, 6 groups were analyzed by oncogenic HR-HPV genotypes. The Alpha-9 (64.8\%, $\mathrm{n}=118),-7(14.3 \%, \mathrm{n}=26)$, and $-5,6$ $(20.9 \%, n=38)$ groups by HPV DNA testing and the Alpha-9 (70.5\%, $\mathrm{n}=110),-7(10.3 \%, \mathrm{n}=16)$, and $-5,6$ 


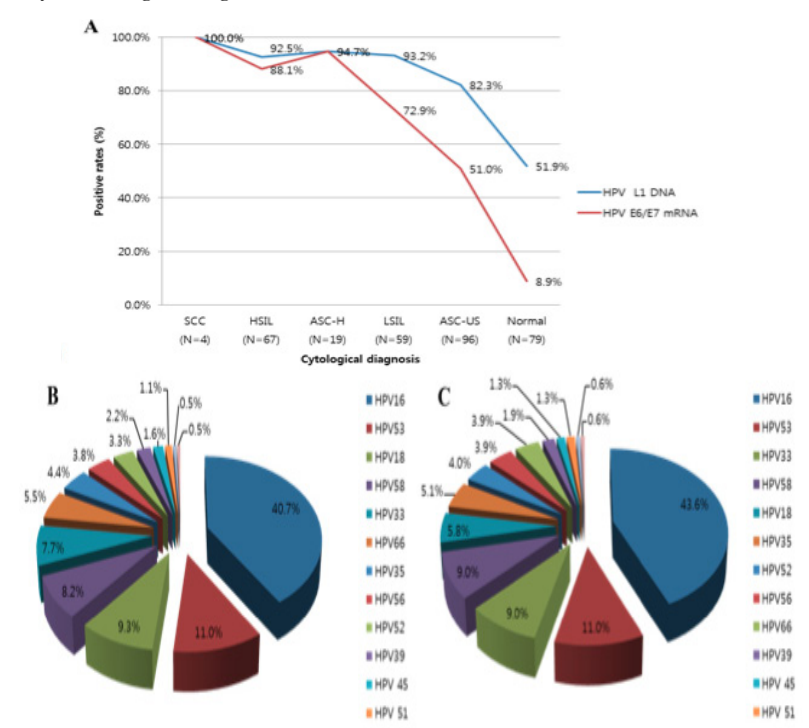

Figure 2. HPV Positivity Rates from HPV L1 DNA and HPV E6/E7 mRNA Assays, According to Cytological Grade (A) and the distribution of oncogenic HR-HPV

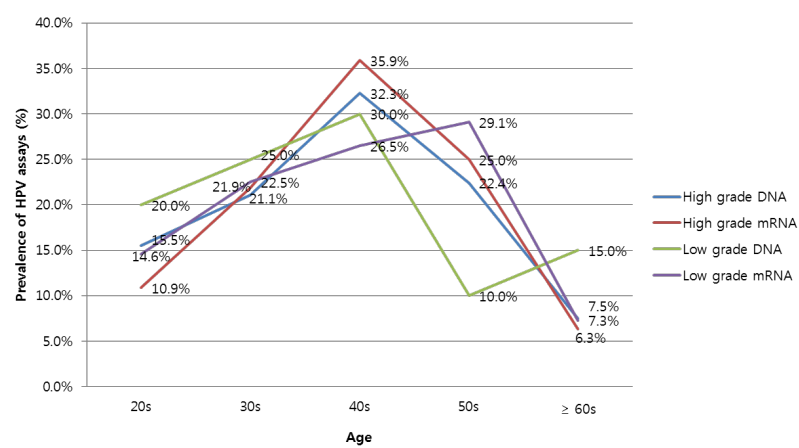

Figure 3. HR-HPV Prevalence Determined by Oncogenic HR-HPV DNA and HR-HPV E6/E7 mRNA Assays among Women with Different Cytological Grades (High - Low), According to Patient Age. Pearson correlation analyzed comparisons between age groups in high grade and low grade specimens, respectively $(\mathrm{p}<0.001)$.

$(19.2 \%, \mathrm{n}=30)$ groups by E6/E7 mRNA testing accounted for the majority of oncogenic HR-HPV genotypes, respectively. Among the oncogenic HR-HPV DNA genotypes, the most common genotype was HPV 16 $(n=74,40.7 \%)$, followed by HPV $53(n=20,11.0 \%)$, HPV $18(\mathrm{n}=17,9.3 \%)$, HPV $58(\mathrm{n}=15,8.2 \%), \operatorname{HPV} 33(\mathrm{n}=14$, 7.7\%), HPV $66(n=10,5.5 \%)$, HPV $35(n=8,4.4 \%)$, HPV $56(\mathrm{n}=7,3.8 \%)$, HPV $52(\mathrm{n}=6,3.3 \%)$, HPV $39(\mathrm{n}=4$, $2.2 \%)$, HPV $45(n=3,1.6 \%)$, HPV $51(n=2,1.1 \%)$, HPV 31 $(\mathrm{n}=1,0.5 \%)$, and HPV $69(\mathrm{n}=1,0.5 \%)$ (Figure 2B). Among the oncogenic HR-HPV E6/E7 mRNA genotypes, the most common genotype was HPV $16(n=68,43.6 \%)$, followed by HPV $53(\mathrm{n}=17,11.0 \%)$, HPV $33(\mathrm{n}=14,9.0 \%)$, HPV 58 $(n=14,9.0 \%)$, HPV $18(n=9,5.8 \%)$, HPV $35(n=8,5.1 \%)$, HPV $56(n=6,3.9 \%)$, HPV $66(n=6,3.9 \%)$, HPV $52(n=5$, $4.0 \%), \operatorname{HPV} 39(\mathrm{n}=3,1.9 \%)$, HPV $45(\mathrm{n}=2,1.3 \%), \mathrm{HPV}$ $51(\mathrm{n}=2,1.3 \% \%), \operatorname{HPV} 31(\mathrm{n}=1,0.6 \%)$, and HPV $69(\mathrm{n}=1$, $0.6 \%)$. HPV 59 and HPV 68 were not detected in the HPV DNA and E6/E7 mRNA assays (Figure 2C).

Distribution of the HR-HPV genotypes based on E6/E7 mRNA expression according to age

The HR-HPV genotype distributions based on HPV
DNA and E6/E7 mRNA expression, assessed according to age group in patients with cytological lesions, was highest in women aged 40 to 49 with high-grade lesions (32.3\% vs. 35.9\%). However, HPV DNA and E6/E7 mRNA showed the highest rate of infection in women aged 40 to 49 years $(30.0 \%)$ and 50 to 59 years $(29.1 \%)$ with low-grade lesions, respectively (Figure 3 ). With respect to the overall distribution of HPV DNA and E6/E7 mRNA based on cytological diagnosis, the Alpha-9 group (118 $(64.8 \%)$ cases vs. $110(70.5 \%)$ cases) was determined to have the highest rate of infection in women of all ages, followed by the Alpha-5, 6 group (38 (20.9\%) cases $v s$. $30(19.2 \%)$ cases), and Alpha-7 group (26 (14.3\%) cases vs. $16(10.3 \%)$ cases $)$. In women under age 30, the most prevalent HPV genotypes detected were HPV 16 (15.5\%), followed by HPV 58, 53, and 56 (3.2\%), 33 (2.6\%), 66 $(1.9 \%), 45,51(1.2 \%)$, and $69(0.6 \%)$. Among women aged 40 or over, the most prevalent HPV genotypes detected were HPV $16(28.4 \%), 53(7.7 \%), 33$ (6.5\%), 58 (5.8\%), $18(4.5 \%), 35(3.2 \%), 18(4.4 \%), 52(2.6 \%), 39,66(1.9 \%)$, $31(3.2 \%)$, and $31,56(0.6 \%)$, respectively (Table 2$)$.

\section{Discussion}

Currently, the combined use of cervical cytology and HPV DNA testing is the mainstay of cervical cancer screening programs. Studies comparing the performance of cervical cytology with HR-HPV DNA detection for the detection of cervical cancer lesions have shown that the Pap smear lacks the sensitivity to detect pre-cervical cancer or cervical cancer lesions in all women, despite the fact that the specificity of the Pap smear is greater than 90\% (Mayrand et al., 2007; Cox, 2009). In contrast, although the sensitivity of high risk HPV DNA is superior to that of cytology for the detection of pre-cervical cancer or cervical cancer, the low specificity of these assays leads to false-positive results (Ronco et al., 2008). Here, our study aimed to evaluate the performance of a new RNA approach to cervical cancer screening which greatly improves specificity. While infection with high-risk HPV is a necessary biological factor for cervical cancer, the actual oncogenic process is initiated by persistent high-risk HPV infection and mediated by the upregulation of the E6/ E7 oncoproteins (Sotlar et al., 2004). Thus, overexpression of these oncoproteins is associated with an increased risk of lesion progression (Münger et al., 2004). Continuous expression of the HR-HPV E6 and E7 oncoproteins is necessary for transformation of normal cells to dysplastic cells (Doorbar, 2006). On this basis, it would stand to reason that the detection of E6/E7 oncogene activity should be more specific and should also be a better predictor of cervical cancer risk than HPV DNA detection methods (Molden et al., 2005). In this study, we found that positivity for HPV E6/E7 mRNA expression was significantly lower than HPV DNA positivity, detecting an overall HPV prevalence of $66.4 \%$ (vs. $79.9 \%$ with the HPV DNA test) when unclassified types were included and $48.1 \%$ (vs. 56.2\% with the HPV DNA test) when only the 16 specific HR-HPV genotypes that are typically tested for were utilized. This difference was especially evident 
in clinically negative, cytologically low grade lesions and in normal samples, in concordance with the results of a previous study (Coquillard et al., 2011). Our results showed that the HPV DNA and HPV E6/E7 mRNA assays were positive in 175/234 (74.8\%) and 99/234 (42.3\%) low grade lesions, respectively. Also, HPV DNA testing was positive in $41 / 79(51.9 \%)$ normal cytology samples whereas the HPV E6/E7 mRNA assay was positive in only $9 / 79(11.4 \%)$ of those samples. Although they were limited in our study and more follow-up studies will be needed on low grade lesions that are HPV DNA positive but HPV mRNA negative, the HPV E6/E7 mRNA assay did more to improve specificity than HPV DNA assay did. Also, the overall concordance rate between the HPV DNA and HPV E6/E7 mRNA assays in our study was 78.4\% and we were able to demonstrate statistically significant differences between these two assays (Pearson correlation $\mathrm{r}$ value $=0.572, \mathrm{p}<0.001)$. The sensitivity and negative predictive values were higher for the HPV-DNA assay (83.0\% and $80.7 \%$, respectively) than for the HPV E6/ E7 mRNA assay (76.5\% and $73.6 \%$, respectively). In contrast, the mRNA test showed higher specificity and positive predictive values $(80.7 \%$ and $83.0 \%$, respectively) than the DNA test $(71.8 \%$ and $76.5 \%$, respectively) for the detection of cytology-confirmed lesions (Pearson correlation $r$ value $=0.620, p<0.001)$. However, the sensitivity of the HPV E6/E7 mRNA assay was higher than the HPV-DNA assay in high grade lesions $(88.0 \%$ vs. $85.9 \%$ ). The expression levels of HPV E6 and E7 mRNA have been found to increase with lesion severity; therefore, the detection of HPV E6/E7 mRNA expression might be of greater prognostic value and might improve the specificity and positive predictive value compared with the HPV DNA testing used in traditional cervical cancer screening.

The purposes of the present study were to identify the prevalence of type-specific HR-HPV genotypes based on E6/E7 mRNA expression compared to the HPV DNA test and to evaluate the association of different HPV genotypes among women with cervical dysplasia. The HPV genotype-distribution data could provide useful information for establishing the appropriate vaccination program, and for creating a diagnostic and treatment strategy for cervical cancer. In previous studies, HPV 16, $18,31,33$, and 45 were reported to be the most prevalent HPV genotypes associated with cervical cancer in Europe (Walboomers et al., 1999; Poljak et al., 2013); HPV 16, 18, 45, 31, and 33 in North America (Bruni et al., 2010); and HPV 16, 18, 45, 33, and 31 in Western and Central Asia (Li et al., 2011); HPV 16, 18, 33, 53, 56, and 58 in Korea (Lee et al., 2011; Kim et al., 2012); and HPV 16, 18, 33, 52, and 58 in Beijing (Ding et al., 2014; Sun et al., 2014). In this study, HPV 16, 53, 18, 58, and 33 were the most prevalent HPV genotypes identified by HPV DNA test and HPV E6/E7 mRNA test in Shandong. Therefore, the HPV genotype-distribution between Eastern Asia and other continents differs greatly (Li et al., 2011). The prevalences of three of the HR-HPV genotypes (HPV $16,18,33)$ seem to be similar in Shandong as to those reported in many other countries, while two HR-HPV genotypes (HPV 53,58) appeared mainly in Shandong. In particular, HPV 53 was more prevalent, whereas HPV 31 and 45 were less prevalent than in studies that looked at women from different countries. Additionally, our results showed that Alpha-9 group genotypes were found more frequently in high grade lesions. This suggests that the Alpha-9 genotypes contribute to oncogenesis in high grade lesions. However, since other HR-HPV genotypes can also potentially promote oncogenesis, testing for the Alpha-7 and Alpha-5, 6 genotypes still needs to be performed.

The overall prevalence of HPV infection and the distribution of HPV genotypes vary with patient age, cytology stage, and geographic region including regions within a single country. Therefore, data describing HPV prevalence by age can be used for early diagnoses. Other studies have shown that HPV positivity is the highest among women less than 20 years of age (just after beginning sexual relations) (Kjaer et al., 2008; Agarossi et al., 2009). However, results from this study were not statistically significant for women younger than 20 years old because samples in this age group were absent. The HR-HPV genotype distributions based on HPV DNA and E6/E7 mRNA expression by age group in patients with cytologically confirmed lesions was highest rate in women aged 40 to 49 years (35.9\% for cytologically confirmed, Pearson correlation $r$ value $=0.993, p<0.001$ ) with high grade lesions, and in those aged 50 to 59 years ( $29.1 \%$, Pearson correlation $\mathrm{r}$ value $=0.925, \mathrm{p}<0.001)$ with low-grade lesions. There was a marked slight increase in the prevalence of HR-HPV with age, both overall (58\% (29/50) of women less than 30 years and $67.4 \%$ (186/276) of women age 30 years or above) and for each HPV genotypes. The age-specific HPV prevalence rates ranged between $0 \%$ and $35.9 \%$ and the patterns of age group specific prevalence, as measured by HPV E6/E7 mRNA, were similar to those of HPV DNA. Among the oncogenic HR-HPV genotypes in all age groups, there was little difference in the distribution of HPV genotypes between the HPV DNA (HPV -16, 53, 18, 58, and 33) and HPV E6/E7 mRNA (HPV -16, 53, 33, 58, and 18) assays.

There are a few limitations to this study. There were a limited number of histologically confirmed CIN 2+ or CIN1 samples for HPV type distribution compared to other studies because our participants were all selected from a single clinic site. Therefore, it will be necessary to conduct more tests with a larger number of histologically confirmed CIN 2+ or CIN1 samples.

In conclusion, our data suggest that HPV E6/E7 mRNA testing may be a useful tool in the investigation of, as well as in primary screening for, cervical cancer. Based on our findings, it might be worthwhile to consider which genotypes to include in future investigations to optimize sensitivity and specificity. The vaccine-targeted HPV 16 and 18 are the most frequent HPV genotypes worldwide and have also been shown to be associated with uterine cervical cancer. However, the extent to which vaccines directed against HPV 16 and 18 would prevent disease associated with other HPV genotypes is not yet clear. The findings of our study suggest that a preventive vaccine against HPV 16 and HPV 18 is not optimal for populations in Eastern China. Based on the results of our study, it is likely that if a preventive vaccine were to include HPV 53 
Hye-Young Wang et al

and HPV 58, the vaccine efficacy could be increased in this province. These data may provide guidance for national or regional vaccination programs in this province aimed at substantially reducing the burden of cervical lesions and HPV infections. The differences in HPV prevalence and genotype distributions identified in this study could potentially influence the effectiveness of HPV vaccines for cervical cancer and the development of screening programs, which should be investigated in future studies.

\section{Acknowledgements}

This research was supported by Basic Science Research Program through the National Research Foundation of Korea (NRF) funded by the Ministry of Science, ICT and future Planning (2015R1A2A2A04004455) and the BK21 Plus Project, Ministry of Education, Republic of Korea (22A20130000035).

\section{References}

Agarossi A, Ferrazzi E, Parazzini F, et al (2009). Prevalence and type distribution of high-risk human papillomavirus infection in women undergoing voluntary cervical cancer screening in Italy. J Med Virol, 81, 529-35.

Andersson E, Karrberg C, Radberg T, et al (2011). Type-specific human papillomavirus E6/E7 mRNA detection by real-time PCR improves identification of cervical neoplasia. J Clin Microbiol, 49, 3794-99.

Ault KA (2007). Human papillomavirus vaccines and the potential for cross-protection between related HPV types. Gynecol Oncol, 107, 31-33.

Bruni L, Diaz M, Castellsague X, et al (2010). Cervical human papillomavirus prevalence in 5 continents: meta-analysis of 1 million women with normal cytological findings. J Infect Dis, 202, 1789-99.

Clifford GM, Gallus S, Herrero R, et al (2005). Worldwide distribution of human papillomavirus types in cytologically normal women in the international agency for research on cancer HPV prevalence surveys: a pooled analysis. Lancet, 366, 991-8.

Coquillard G, Palao B, Patterson BK (2011) Quantification of intracellular HPV E6/E7 mRNA expression increases the specificity and positive predictive value of cervical cancer screening compared to HPV DNA. Gynecologic Oncol, 120, 89-93.

Cox, JT (2009). History of the use ofHPV testing in cervical screening and in the management of abnormal cervical screening results. J Clin Virol, 45, 3-12.

de Sanjose S, Serrano B, Castellsague X, et al (2012). Human papillomavirus (HPV) and related cancers in the global alliance for vaccines and immunization (GAVI) countries. A WHO/ICO HPV information centre report. Vaccine, 30, 1-83.

Ding X, Liu Z, Su J, et al (2014). Human papillomavirus typespecific prevalence in women referred for colposcopic examination in Beijing. J Med Virol, 86, 1937-43.

Doorbar J (2006). Molecular biology of human papillomavirus infection and cervical cancer. Clinical science, 110, 525-41.

Ergünay K, Misirlioglu M, Pinar F, et al (2007). Human papilloma virus DNA in cervical samples with cytological abnormalities and typing of the virus. Mikrobiyol Bul, 41, 219-26.

Jemal A, Bray F, Center MM, et al (2011). Global cancer statistics. CA Cancer J Clin, 61, 69-90.

Kim S, Lee D, Park S, et al (2012). REBA HPV-ID ${ }^{\circledR}$ for efficient genotyping of human papillomavirus in clinical samples from Korean patients. J Med Virol, 84, 1248-53.

Kjaer SK, Breugelmans G, Munk C, et al (2008). Populationbased prevalence, type- and age-specific distribution of HPV in women before introduction of an HPV-vaccination program in Denmark. Int J Cancer, 123, 1864-70.

Laudadio, J (2013). Human papillomavirus detection: testing methodologies and their clinical utility in cervical cancer screening. Adv Anat Pathol, 20, 158-67.

Lee, D, Kim S, Park S, et al (2011). Human papillomavirus prevalence in gangwon province using reverse blot hybridization assay. Korean J Pathol, 45, 348-53.

Li N, Franceschi S, Howell-Jones R, et al (2011). Human papillomavirus type distribution in 30,848 invasive cervical cancers worldwide: Variation by geographical region, histological type and year of publication. Int J Cancer, 128, 927-35.

Liu XX, Fan XL, Yu YP, et al (2014). Human papillomavirus prevalence and type-distribution among women in Zhejiang province, southeast china: across-sectional study. BMC Infect Dis, 14, 708-14.

Mayrand MH, Duarte-Franco E, Rodrigues I, et al (2007). Human papillomavirus DNA versus papanicolaou screening tests for cervical cancer. N Engl J Med, 357, 1579-88.

Molden T, Kraus I, Karlsen F, et al (2005). Comparison of human papillomavirus messenger RNA and DNA detection: a cross sectional study of 4,136 women $>30$ years of age with a 2-year follow-up of high-grade squamous intraepithelial lesion. Cancer Epidemiol Biomarkers Prev, 14, 367-72.

Münger K, Baldwin A, Edwards KM, et al (2004). Mechanisms of human papillomavirus-induced oncogenesis. J Virol, 78, 11451-60.

Okadome M, Saito T, Tanaka H, et al (2014). Potential impact of combined high- and low-risk human papillomavirus infection on the progression of cervical intraepithelial neoplasia 2. J Obstet Gynaecol Res, 40, 561-69.

Poljak M, Seme K, Maver PJ, et al (2013). Human papillomavirus prevalence and type-distribution, cervical cancer screening practices and current status of vaccination implementation in Central and Eastern Europe. Vaccine, 7, 59-70.

Ronco G, Giorgi-Rossi P, Carozzi F, et al (2008). New technologies for cervical cancer screening working group. Results at recruitment from a randomized controlled trial comparing human papillomavirus testing alone with conventional cytology as the primary cervical cancer screening test. J Natl Cancer Inst, 100, 492-501.

Sotlar K, Stubner A, Diemer D, et al (2004). Detection of highrisk human papillomavirus E6 and E7 oncogene transcripts in cervical scrapes by nested RT-polymerase chain reaction. J Med Virol, 74, 107-16.

Sun B, He J, Chen X, et al (2014). Prevalence and genotype distribution of human papillomavirus infection in harbin, Northeast China. Arch Virol, 159, 1027-32.

Sørbye SW, Fismen S, Gutteberg T, et al (2010). Triage of women with minor cervical lesions: data suggesting a "test and treat" approach for HPV E6/E7 mRNA testing. PLoS One, 5, 12724-29.

Vizcaino AP, Moreno V, Bosch FX, et al (1998). International trends in the incidence of cervical cancer: I. Adenocarcinoma and adenosquamous cell carcinomas. Int J Cancer, $\mathbf{7 5}$, 536-45.

Walboomers JM, Jacobs MV, Manos MM, et al (1999). Human papillomavirus is a necessary cause of invasive cervical cancer worldwide. J Pathol, 189, 12-19.

zur Hausen, H (2009). Papillomaviruses in the causation of human cancers - a brief historical account. Virology, 384, 260-65. 